50 patients of investigational group (IG) additionally were treated by Sibutramin, daily dosage contained $15 \mathrm{mg}$ and PUPC (daily dosage contained $1368 \mathrm{mg}$ Phosphatidylcholine). 30 patients of control group (CG) were treated by Sibutramin, daily dosage contained $15 \mathrm{mg}$.

Results All 50 patients of IG and 30 patients of CG were available for follow-up reliable decrease (12.8\%) of BMI and leptin level. In IG patients BMI and leptin level $(24.7 \pm 2.1 \%-16.2 \pm 1.2 \%)$ was registered compared to CG patients (BMI decrease-9.3\%, leptin level $24.5 \pm 1.5 \%$ to $19.9 \pm 1.1 \%$ ). Moreover after 6 months of treatment the mean value of disease activity evaluated by Metavir scale was A1 in IG and A3 in CG. The results of liver biopsy (histological examination) and Fibromax test showed, that in patients with ALD additionally treated by PUPC, the progress of hepatic fibrosis was significantly slowly, then in CG (Fibromax test result: F1 vs F3) $(p<0.05)$. In addition after 6 months of treatment we found significant increase of steatosis in CG, and its reduction in IG $(p<0.05)$. Ultrasound studies were performed on the basis of liver attenuation value assessment and revealed the hepatic echotexture had become significantly improved after Sibutramin treatment in $46 / 50$ (92\%) of patients, while there was no change in $7 / 30$ $(23.3 \%)$ individuals. Also in patients additionally treated by polyunsaturated phosphatidylcholine and Sibutramin we found significant reduction of glucose, insulin levels and of insulin resistance index HOMA. The usage of Sibutramin itself did not reveal significant changes.

Conclusion Study results suggest that Sibutramin combined with PUPC improves liver function and insulin-sensitivity, reduces steatosis and fibrosis in patients with obesity and effect of combined therapy is higher than of Sibutramin itself.

Competing interests None declared.

\section{PTU-081 TREATMENT AND SURVEILLANCE OF POLYPOID LESIONS OF THE GALLBLADDER IN THE UK: RESULT OF A SURVEY}

doi:10.1136/gutjnl-2012-302514c.81

G Marangoni, * A Hakeem, G J Toogood, J P A Lodge, R K Prasad. HPB and Transplant Unit, St James' Hospital, Leeds, UK

Introduction The management of gallbladder polyps (GBP) is still controversial. The increased use of routine abdominal imaging has led to a parallel surge of identified polypoid lesions in the gallbladder. The vast majority of these lesions are benign. True polyps, which are less frequent, have a malignant potential and surgery can prevent/ treat early gallbladder cancer. In an era of constraint on health care resources it is important to offer cholecystectomy only to patients who have appropriate indications.

Methods The aim of this study was to assess the treatment and surveillance policies of GBP among hepatobiliary and upper GI surgeons in UK in the light of the current published literature. A questionnaire on GBP was devised and sent to the Consultant Surgeon members of the Association of Upper GI Surgeons (AUGIS) of Great Britain and Ireland after approval from the AUGIS Committee. There were eight questions regarding indications for laparoscopic cholecystectomy (LC) and surveillance based on GBP characteristics (size, number and growth rate), and patient characteristics (age, comorbidities and ethnicity).

Results There were 79 completed questionnaires. Three-quarters of surgeons consider $1 \mathrm{~cm}$ as the size threshold for recommending surgery but $9 \%$ would consider LC irrespective of GBP size. $25 \%$ would recommend LC for multiple polyps irrespective of the size of the largest GBP. $28 \%$ of surgeons emphasise a growth rate of $5 \mathrm{~mm}$ or more as an indication for LC; more than $50 \%$ would not offer LC unless the polyp size matches their criteria for single polyp LC. $25 \%$ would recommend surgery for any number increase of GBP between surveillance scans. Surveillance protocols were heterogeneous but about $40 \%$ would agree to surveillance up to 5 years. About $30 \%$ would not offer LC for octagenarians and 10\% would reconsider their surgery treshold according to ethnicity.

Conclusion GBP are a relatively common finding on abdominal ultrasound scans. About 50000 LC are performed each year in UK and 800-4000 are for GBP. The survey has shown considerable heterogeneity among surgeons regarding treatment and surveillance protocols. Although no randomised controlled trials exist, international guidelines would help standardisation, formulation of an appropriate algorithm and appropriate use of resources.

Competing interests None declared.

\section{PTU-082 RIGHT AND EXTENDED RIGHT HEPATIC TRISECTIONECTOMY: SHORT AND LONG TERM OUTCOMES OF 332 RESECTIONS}

doi:10.1136/gutjnl-2012-302514c.82

G Marangoni, * A Hakeem, R Storey, Z Hamady, H Sethi, G J Toogood, R K Prasad, J P A Lodge. HPB and Transplant Unit, St James' Hospital, Leeds, UK

Introduction Despite advances in surgical and anaesthetic techniques made over the last 2 decades, right hepatic trisectionectomy (RHT) is still a challenging procedure associated with higher rates of morbidity and mortality. Some patients may even require further extension of the resection to include part of segments II/III to achieve clearance (extended right hepatic trisectionectomy, ERHT). Aim of the study was to assess and compare the early and long-term outcomes of RHT and ERHT in our Unit.

Methods From January 1993 to December 2010, 252 RHT and 80 ERHT were performed $(n=332)$. Resection for colorectal liver metastases (CRLM), HCC, cholangiocarcinoma and other were 127, 43, 25 and 57 for RHT and 60, 3, 2, 15 for ERHT respectively. Mean age was 58.3 vs 57.9 and $57.1 \%$ vs $55 \%$ were males (RHT vs ERHT, $\mathrm{p}=\mathrm{NS}$ ). There were 61 caudatectomy in the RHT group and 15 in the ERHT ( $p=0.36, N S)$; vascular resection (IVC or PV) was performed in 61 and 10 cases $(p=0.18, N S)$, biliary reconstruction was performed in 75 and 7 cases $(p=0.01)$ and total vascular exclusion was necessary in 26 and 6 cases respectively $(p=N S)$. The amount of functional hepatic remnant was based on intra-operative judgement.

Results There were 23 in-hospital deaths (6.9\%, RHT: 19, ERHT: 4; $\mathrm{p}=\mathrm{NS}$ ). Overall morbidity was $44 \%$ (RHT) and $47.5 \%$ (ERHT). Bile leak (17 vs 3 ), haemorrhage (14 vs 4), sepsis (33 vs 9), cardiovascular events (12 vs 1 ) and renal failure (12 vs 3 ) did not differ among the two groups (RHT vs ERHT; $p=N S$ ). There were 42 $(12.6 \%)$ post-hepatectomy liver failure (according to "50:50 criteria"): 23 in the RHT group and 19 in the ERHT group respectively ( $p=0.001$ ). Mean hospital stay was 15.8 vs 17.1 days (RHT vs ERHT, $\mathrm{p}=\mathrm{NS}$ ). In the CRLM cohort, 1,5 and 10 year survival was $78 \%, 47 \%$ and $39 \%$ vs $79 \%, 47 \%$ and $37 \%$ (RHT vs ERHT, $\mathrm{p}=0.93$, NS). Median survival was 49 and 43.9 months respectively ( $\mathrm{p}=\mathrm{NS}$ ) and median follow-up was 59.7 and 56.5 months (RHT vs ERHT).

Conclusion RHT and ERHT are a major undertaking with significant morbidity and mortality but represent the only chance of cure in selected patients. Liver failure is higher in the ERHT group but does not translate in increased mortality. Long-term survival in CRLM is achievable and does not differ among the two groups. Extensive liver resections even beyond conventional boundaries should not be considered an absolute contraindication to surgery.

Competing interests None declared. 